\title{
Chapter 7 \\ Putting the Mouth into Health: \\ The Importance of Oral Health for General Health
}

\author{
Christopher C. Peck
}

\begin{abstract}
Good oral health is important to overall health and wellbeing. From the most fundamental perspectives of the oral-systemic health relationship, good oral health ensures an individual can eat, speak and socialise without pain or embarrassment. These capabilities improve an individual's wellbeing and ability to contribute to society. There are more complex oral-systemic health relationships in which poor oral health can contribute to systemic diseases including the links between orofacial disease and, for example, diabetes, cardiovascular disease, pneumonia or rheumatoid arthritis. Furthermore, the oral environment is a complex biological ecosystem which provides an opportunity to understand mechanisms underlying pathological processes and which can be a model for other body systems. Examples of these oral-systemic interactions are presented to demonstrate the importance of the orofacial environment and the need for it to be incorporated into health more broadly. By putting the mouth into health through integrated education, research and clinical care across the health disciplines, there will be advances in health that will improve individual and community health.
\end{abstract}

Keywords Health • Oral-systemic health • Pain • Disability

\subsection{Introduvction}

The orofacial region is unique because of its importance in social interactions, including communication and emotional expression, and in survival with mastication and lifesaving reflexes that protect other external sensory (e.g. retina, olfactory epithelium, taste receptors) and internal homeostatic (e.g. respiratory and digestive tracts) systems from damaging environmental changes. Consequently this region and good oral health are important components to overall health and wellbeing.

C.C. Peck $(\bowtie)$

Faculty of Dentistry, The University of Sydney, Level 2, G12 - Services Building,

22 Codrington Street, Darlington, NSW 2006, Australia

e-mail: dentistry.dean@sydney.edu.au 
From the most fundamental perspectives of the oral-systemic health relationship, good oral health ensures an individual can eat, speak and socialise without pain or embarrassment. These capabilities improve an individual's wellbeing and ability to contribute to society. There are more complex oral-systemic health relationships in which poor oral health can contribute to systemic diseases including the links between orofacial disease and, for example, diabetes, cardiovascular disease, pneumonia or rheumatoid arthritis. Furthermore, the oral environment is a complex biological ecosystem which provides an opportunity to understand mechanisms underlying pathological processes, and which therefore can be a model for other body systems.

There have been significant advances in oral health improvement over the past 30 years, where there has been a change in focus from the acute management of the disease burden of dental caries and periodontal disease to an increased focus on prevention and more complex oral rehabilitation to improve function and aesthetics. Nevertheless, in Australia as in many parts of the world, there is still a worrying oral disease burden; oral cancer continues with significant morbidity and mortality [1], $30 \%$ of adults have untreated tooth decay [2], $15 \%$ of adults experience oral pain [3], $25 \%$ adults are uncomfortable about their dental appearance [3] and oral problems account for the third highest level of acute preventable hospital admissions [4]. Furthermore they can affect self-esteem, social interaction, education, career achievement and emotional state [5] and lead to a deteriorating diet and compromised nutrition [6].

\subsection{The Oral-Systemic Nexus}

Whilst many countries, including Japan and Australia, have universal healthcare systems, frequently dental care is partly or completely excluded. This leads to societal inequality with groups, such as Aboriginal Australians, who are on low income, with limited education or living in remote areas suffering worse oral health. In a similar way that many governments exclude oral health from healthcare, many individuals, when prioritising household expenditure, consider oral health as a cosmetic issue rather than essential to overall health. In Australia over $45 \%$ of adults over 25 years did not visit a dental practitioner in the past year [7].

There is much research indicating links between oral health and, for example, cardiovascular disease, mental health and diabetes. There are interesting findings showing that a person with fewer than ten of their own teeth is seven times more likely to die of coronary disease than someone with more than 25 of their own teeth [8] and that treating gum disease improves vascular health [9]. With Alzheimer's disease, a study of more than 4000 Japanese participants, aged 65 and older, found those with missing teeth were much more likely to have experienced memory loss or have early-stage Alzheimer's disease than those with an intact dentition [10]. The relationship between diabetes mellitus and periodontal disease appears bidirectional with diabetes increasing the risk for periodontitis and periodontal inflammation 
negatively affecting glycaemic control [11]. The relationship between the two diseases is strong and in fact periodontal disease has been coined the sixth complication of diabetes [12]. These and many other associations between oral and systemic health are important and the focus of much research, particularly exploring aetiological mechanisms underlying these associations.

As a result of the strong links between oral and systemic health, we have embarked on a strategic initiative to put the mouth into health, through education, research and clinical care. Our research endeavours are focussed on oral-systemic health interactions in exploring known associations, using the oral environment as a biomarker and as a model for disease processes or clinical care. Some examples of these endeavours follow.

\subsection{Oral-Systemic Health Associations}

There are strong associations between rheumatoid arthritis and periodontal disease, and it is important to determine if this is because they are two common chronic diseases or indeed if there is a causal link between the two diseases [13]. Interdisciplinary research suggests that the periodontal pathogen Porphyromonas gingivalis exacerbates collagen-induced arthritis (rheumatoid arthritis). The bacteria express an enzyme that causes human and bacterial protein citrullination (conversion of the amino acid arginine into citrulline) and autoantibodies to these citrullinated proteins. A pathogenic autoimmune response to these citrullinated proteins ensues causing earlier onset, accelerated progression and enhanced severity of the arthritic disease [14]. These findings may help with diagnosis and targeted treatments.

Chronic orofacial pain can be disabling affecting approximately $10 \%$ of the adult population [15]. Pain is frequently not limited to the orofacial region; a local survey demonstrated up to $60 \%$ of clinical patients reported pain elsewhere in their body (Fig. 7.1). Furthermore, subjects with temporomandibular disorders frequently had symptoms of widespread conditions including chronic fatigue syndrome and fibromyalgia [16]. We have demonstrated that temporomandibular disorders can be associated with catastrophic beliefs and with depression and that these psychological factors can impact jaw motor activity [17, 18].

Oral health appears to be related to physical health, specifically cardiorespiratory fitness. In a clinical study of 72 men, peak oxygen uptake during exercise testing was significantly lower, indicating lower levels of fitness, in those with moderate to severe periodontitis [19].

In an interesting study comparing genetic details of calcified dental plaque from 34 early European skeletons, it was shown that the transition from hunter-gatherer to farming shifted the oral microbial community to a disease-associated configuration. During the Industrial Revolution, cariogenic bacteria became dominant, and the modern oral microbial community is much less diverse than historic populations [20]. This may be contributing to contemporary chronic diseases, affecting both oral and systemic environments. 


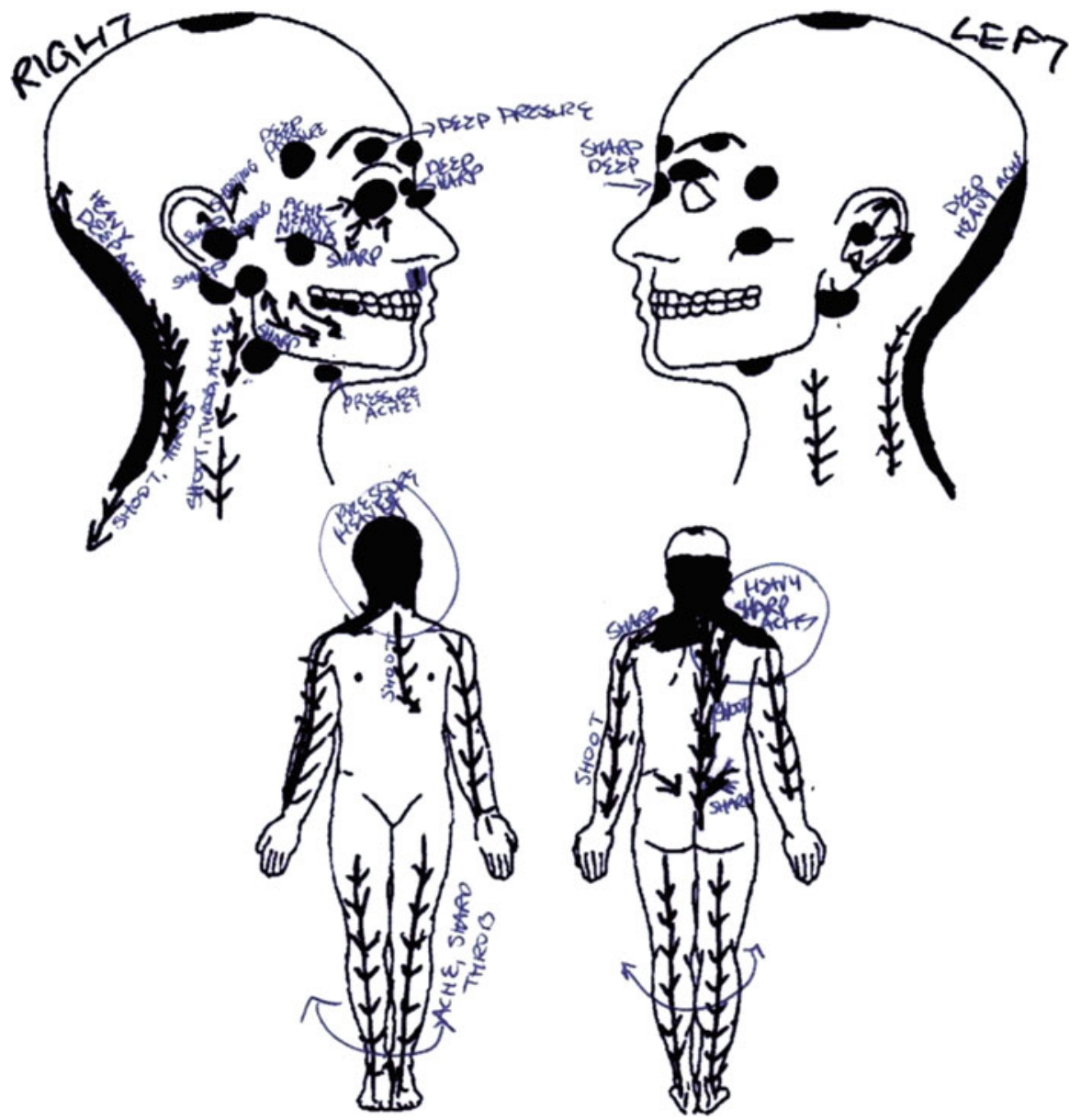

Fig. 7.1 An example of a pain map for an orofacial pain patient demonstrating pain extending beyond the oral and facial regions (shaded regions and arrows located painful regions and pain spread)

\subsection{The Oral Environment: A Model for Other Body Systems}

The oral environment is a complex and harsh biological setting connecting the internal body with the external environment. It consists of hard and soft tissues, biofilm and sensory and motor innervation and consequently can be a good model to monitor systemic health and demonstrate interactions between systems and the external environment. It is important for health professionals and especially dental practitioners to promote the importance of oral health to health overall. 
The tooth can be a biomarker to determine, for example, past chemical exposure during development. One topical area is iron exposure during development in the form of iron-fortified supplements, and the possible relationship with age-related neurodegenerative disorders. Iron has been implicated in disorders such as Parkinson's disorder and it may be the iron levels during development that are implicated. Iron levels in tooth enamel and dentine can be directly mapped to the source and timing of infant nutrition during development of the tooth, thus providing an opportunity to correlate this with neurodegenerative disorders [21]. This retrospective method is attractive for exploring past chemical exposure during the development years. In turn, findings from this will be able to help inform on development of disorders and health policy for nutritional supplements.

Glial cells which make up the majority of cells in the brain were considered previously as scaffolds to support neurons. We now know that these cells' role is much more, and recent work has demonstrated an important role in the dental pulp. With dental caries, and associated hypoxic stress, there is angiogenic remodelling of the pulpal microvasculature through an interaction of endothelial cells, pericytes, microglia and telacytes in the pulp. This response provides vascular remodelling whilst maintaining a blood barrier. The directional expansion of the microvasculature is achieved through glial-assisted guided migration of endothelial cells [22]. This important function can be compared with other glial cell activities in the nervous system.

Temporomandibular disorders are the most common chronic pain disorders in the orofacial region and consist of a number of problems affecting the jaw's musculoskeletal system including the temporomandibular joints or masticatory muscles. The classification scheme for these disorders has been a model for other pain conditions and disorders as it includes both physical and psychosocial dimensions [23]. Recently it has been revised [24] with validated diagnostic criteria and an extended taxonomy has also been developed [25]. This research on temporomandibular disorders spanning 26 years provides a valuable record for developing diagnostic frameworks for other conditions throughout the body.

\subsection{Summary}

It is imperative to put the mouth into health to improve health and wellbeing of the individual and of society. There are many associations between oral and systemic health and research is investigating causal links. Embedding research, education and clinical care across the health disciplines provides opportunities to better understand the role of oral health in a much broader context. 


\section{References}

1. Australian Institute of Health and Welfare. Head and neck cancers in Australia, Cancer series no. 83. Cat. no. CAN 80. Canberra: AIHW; 2014.

2. Roberts-Thomson K, Luzzi L, Brennan D. Social inequality in use of dental services: relief of pain and extractions. Aust N Z J Public Health. 2008;32(5):444-9.

3. Chrisopoulos S, Harford JE. Oral health and dental care in Australia: key facts and figures 2012, Cat. no. DEN 224. Canberra: AIHW; 2013.

4. Australian Institute of Health and Welfare. Australian hospital statistics 2012-13, Health services series no. 54. Cat. no. HSE 145. Canberra: Australian Institute of Health and Welfare; 2014.

5. US Department of Health and Human Services. Oral health in America: a report of the Surgeon General. Rockville: US Department of Health and Human Services, National Institute of Dental and Craniofacial Research, National Institutes of Health; 2000.

6. Locker D. The burden of oral health in a population of older adults. Commun Dent Health. 1992;9(2):109-24.

7. AIHW, Chrisopoulos S, Harford JE, Ellershaw A. Oral health and dental care in Australia: key facts and figures 2015, Cat. no. DEN 229. Canberra: AIHW; 2016.

8. Holmlund A, Holm G, Lind L. Number of teeth as a predictor of cardiovascular mortality in a cohort of 7,674 subjects followed for 12 years. J Periodontol. 2010;81(6):870-6.

9. Kapellas K, Maple-Brown LJ, Jamieson LM, Do LG, O'Dea K, Brown A, Cai TY, Anstey NM, Sullivan DR, Wang H, Celermajer DS, Slade GD, Skilton MR. Effect of periodontal therapy on arterial structure and function among aboriginal Australians: a randomized. Control Trial Hypertens. 2014;64:702-8.

10. Okamoto N, Morikawa M, Okamoto K, Habu N, Iwamoto J, Tomioka K, Saeki K, Yanagi M, Amano N, Kurumatani N. Relationship of tooth loss to mild memory impairment and cognitive impairment: findings from the fujiwara-kyo study. Behav Brain Funct. 2010;6:77.

11. Preshaw PM, Alba AL, Herrera D, Jepsen S, Konstantinidis A, Makrilakis K, Taylor R. Periodontitis and diabetes: a two-way relationship. Diabetologia. 2012;55(1):21-31.

12. Löe H. Periodontal disease: the sixth complication of diabetes mellitus. Diabetes Care. 1993;16:329-34.

13. de Smit MJ, Brouwer E, Vissink A, van Winkelhoff AJ. Rheumatoid arthritis and periodontitis; a possible link via citrullination. Anaerobe. 2011;17:196-200.

14. Maresz KJ, Hellvard A, Sroka A, Adamowicz K, Bielecka E, Koziel J, et al. Porphyromonas gingivalis facilitates the development and progression of destructive arthritis through its unique bacterial Peptidylarginine Deiminase (PAD). PLoS Pathog. 2013;9(9):e1003627.

15. Zakrzewska JM. Diagnosis and management of non-dental orofacial pain. Dent Update. 2007;34:134-6. 138-9.

16. Aaron LA, Burke MM, Buchwald D. Overlapping conditions among patients with chronic fatigue syndrome, fibromyalgia, and temporomandibular disorder. Arch Intern Med. 2000;160(2):221-7.

17. Henderson LA, Akhter R, Youssef AM, Reeves JM, Peck CC, Murray GM, Svensson P. The effects of catastrophizing on central motor activity. Eur J Pain. 2016;20:639-51.

18. Brandini D, Benson J, Nicholas M, Murray G, Peck C. Chewing in temporomandibular disorder patients: an exploratory study of an association with some psychological variables. J Orofac Pain. 2011;25(1):56-67.

19. Eberhard J, Stiesch M, Kerling A, Bara C, Eulert C, Hilfiker-Kleiner D, Hilfiker A, Budde E, Bauersachs $\mathrm{J}$, et al. Moderate and severe periodontitis are independent risk factors associated with low cardiorespiratory fitness in sedentary non-smoking men aged between 45 and 65 years. J Clin Periodontol. 2014;41(1):31-7.

20. Adler C, Dobney K, Weyrich L, Kaidonis J, Walker A, Haak W, Bradshaw C, Townsend G, Soltysiak A, Alt K, et al. Sequencing ancient calcified dental plaque shows change in oral microbiota with dietary shifts of the Neolithic and Industrial revolutions. Nat Genet. 2013;45(4):450-5. 
21. Hare DJ, et al. Is early-life iron exposure critical in neurodegeneration? Nat Rev Neurol. 2015;11(9):536-44. doi:10.1038/nrneurol.2015.100.

22. Farahani RM, Sarrafpour B, Simonian M, Li Q, Hunter N. Directed glia-assisted angiogenesis in a mature neurosensory structure: pericytes mediate an adaptive response in human dental pulp that maintains blood-barrier function. J Comp Neurol. 2012;520:3803-26.

23. Dworkin SF, LeResche L. Research diagnostic criteria for temporomandibular disorders: review, criteria, examinations and specifications, critique. J Craniomandib Disord. 1992;6(4):301-55.

24. Schiffman E, Ohrbach R, Truelove E, et al. Diagnostic Criteria for Temporomandibular Disorders (DC/TMD) for clinical and research applications: recommendations of the International RDC/TMD Consortium Network and Orofacial Pain Special Interest Group. J Oral Facial Pain Headache. 2014;28(1):6-27.

25. Peck CC, Goulet J-P, Lobbezoo F, et al. Expanding the taxonomy of the diagnostic criteria for temporomandibular disorders (DC/TMD). J Oral Rehabil. 2014;41(1):2-23.

Open Access This chapter is distributed under the terms of the Creative Commons Attribution 4.0 International License (http://creativecommons.org/licenses/by/4.0/), which permits use, duplication, adaptation, distribution and reproduction in any medium or format, as long as you give appropriate credit to the original author(s) and the source, provide a link to the Creative Commons license and indicate if changes were made.

The images or other third party material in this chapter are included in the work's Creative Commons license, unless indicated otherwise in the credit line; if such material is not included in the work's Creative Commons license and the respective action is not permitted by statutory regulation, users will need to obtain permission from the license holder to duplicate, adapt or reproduce the material. 